\title{
Specification Testing of Production in a Stochastic Frontier Model \\ Xu Guo
}

Complutense de Análisis Económico
School of Statistics, Beijing Normal University, Beijing

\section{Gao-Rong Li}

Beijing Institute for Scientific and Engineering Computing, Beijing University of Technology, Beijing

\section{Michael McAleer}

Department of Quantitative Finance, National Tsing Hua University

Discipline of Business Analytics, University of Sydney Business School

Econometric Institute, Erasmus School of Economics, Erasmus University Rotterdam

Department of Quantitative Economics, Complutense University of Madrid

\section{Wing-Keung Wong}

Department of Finance and Big Data Research Center, Asia University

Department of Economics and Finance, Hang Seng Management College

Department of Economics, Lingnan University

\begin{abstract}
Parametric production frontier functions are frequently used in stochastic frontier models, but there do not seem to be any empirical test statistics for its plausibility. To bridge the gap in the literature, we develop two test statistics based on local smoothing and an empirical process, respectively. Residual-based wild bootstrap versions of these two test statistics are also suggested. The distributions of technical inefficiency and the noise term are not specified, which allows specification testing of the production frontier function even under heteroscedasticity. Simulation studies and a real data example are presented to examine the finite sample sizes and powers of the test statistics. The theory developed in this paper is useful for production mangers in their decisions on production.
\end{abstract}

Keywords Production frontier function; Stochastic frontier model; Specification testing; Wild bootstrap; Smoothing process; Empirical process; Simulations.

JEL Classification C0, C13, C14, D81

\section{Working Paper no 1723 October, 2017}




\title{
Specification Testing of Production in a Stochastic Frontier Model
}

\author{
Xu Guo ${ }^{1}$, Gao-Rong Li $^{2}$, Michael McAleer ${ }^{3,4,5,6}$, Wing-Keung Wong ${ }^{7,8,9 *}$ \\ ${ }^{1}$ School of Statistics, Beijing Normal University, Beijing \\ ${ }^{2}$ Beijing Institute for Scientific and Engineering Computing, \\ Beijing University of Technology, Beijing \\ ${ }^{3}$ Department of Quantitative Finance, National Tsing Hua University \\ ${ }^{4}$ Discipline of Business Analytics, University of Sydney Business School \\ ${ }^{5}$ Econometric Institute, Erasmus School of Economics, Erasmus University Rotterdam \\ ${ }^{6}$ Department of Quantitative Economics, Complutense University of Madrid \\ 7 Department of Finance and Big Data Research Center, Asia University \\ ${ }^{8}$ Department of Economics and Finance, Hang Seng Management College \\ ${ }^{9}$ Department of Economics, Lingnan University
}

\begin{abstract}
${ }^{*}$ Corresponding author: Wing-Keung Wong; Email: wong@asia.edu.tw. The fourth author would like to thank Robert B. Miller and Howard E. Thompson for their continuous guidance and encouragement. The research is partially supported by National Tsing Hua University, University of Sydney Business School, Erasmus School of Economics, Erasmus University Rotterdam, Complutense University of Madrid, Asia University, Hang Seng Management College, Lingnan University, Fundamental Research Funds for the Central Universities, China Postdoctoral Science Foundation (2017M610058), Natural Science Foundation of China (11626130, 11601227), Australian Research Council, and Natural Science Council, Taiwan, Ministry of Science and Technology (MOST), Taiwan, and Research Grants Council (RGC) of Hong Kong.
\end{abstract}




\title{
Specification Testing of Production in a Stochastic Frontier Model
}

\begin{abstract}
Parametric production frontier functions are frequently used in stochastic frontier models, but there do not seem to be any empirical test statistics for its plausibility. To bridge the gap in the literature, we develop two test statistics based on local smoothing and an empirical process, respectively. Residual-based wild bootstrap versions of these two test statistics are also suggested. The distributions of technical inefficiency and the noise term are not specified, which allows specification testing of the production frontier function even under heteroscedasticity. Simulation studies and a real data example are presented to examine the finite sample sizes and powers of the test statistics. The theory developed in this paper is useful for production mangers in their decisions on production.

Keywords: Production frontier function; Stochastic frontier model; Specification testing; Wild bootstrap; Smoothing process; Empirical process; Simulations.
\end{abstract}

JEL Classification : C0, C13, C14, D81 


\section{Introduction}

Since the seminal work of Aigner et al. (1977) and Meeusen and van den Broeck (1977), stochastic frontier analysis (SFA) has been a very appealing and popular approach for studying productivity and efficiency analysis. Greene (1990) extends the stochastic frontier model by allowing the one-sided component of the disturbance to have a two-parameter Gamma distribution rather than the less flexible half-normal distribution. Greene (2005) extends the model further by using a nonlinear specification. For an up-to-date introduction and literature review, see Kumbhakar and Lovell (2000) and Fried et al. (2008).

Consider the following SFA model:

$$
Y=m(X)-U+V
$$

where $Y$ is the $\log$ of output, $X$ is the $\log$ of inputs of dimension $p, m(\cdot)$ is an unknown smooth production frontier function, $U$ is the inefficiency term, and $V$ represents random noise. Assume that the positive random variable, $U$, and the symmetric noise term, $V$, are conditionally independent, given the inputs $X$, and $E(V \mid X)=0$.

Parametric SFA models specify the functional form of the production frontier function, $m(\cdot)$, as well as the distributions of the inefficiency term, $U$, and the independent noise, $V$. A fully parametric SFA framework sacrifices 
flexibility, and has been criticized as a major deficiency of SFA models (see Simar and Wilson (2015)).

On the one hand, some authors have discussed how to test the distributional assumptions on $U$ and/or $V$. For instance, Wang et al. (2011) develop the Pearson $\chi^{2}$ and Kolmogorov-Smirnov tests for the distribution of $U$. Chen and Wang (2012) propose a centered residuals-based method of moments to test the distributional assumptions on both $U$ and $V$ (see also Schmidt and Lin (1984), Coelli (1995), Lee (1983), and Kopp and Mullahy (1990)). However, it should be noted that all these procedures are based on the assumed parametric form of the production frontier function. If the parametric assumption on $m(\cdot)$ is not valid, the conclusions can be inaccurate and misleading.

On the other hand, there have been attempts to reduce the parametric restrictions on the production frontier function. Fan et al. (1996) introduce the quasi-likelihood method, where the production frontier is not specified, but distributional assumptions are imposed on the stochastic components. Kumbhakar et al. (2007) propose a local maximum likelihood method but without parametric assumptions on the production frontier function, while using semi-parametric assumptions about $U$ and $V$.

Recently, Simar et al. (2016) develop a nonparametric least squares method to avoid the high computational complexity involved in the local 
maximum likelihood method in Kumbhakar et al. (2007). Another merit of Simar et al. (2016) is that only local distributional assumptions on $U$ are needed, although symmetry is still necessary for $V$. Nonetheless, it should be realized that, if the hypothetical parametric model is satisfied, the methods discussed above would not be necessary. Studying the "wrong skewness phenomenon' in stochastic frontiers (SF), Bonanno et al. (2017) propose a more general and flexible specification of the SF model by introducing dependences between the two error components and asymmetry of the random error.

These studies above call for the specification testing of the production frontier function. Parametric specifications for the frontier are appealing because they offer easy economic interpretation of the production process. Furthermore, due to well established theories, easy computation and interpretation, parametric SFA models have been dominant in the area of productivity and efficiency analysis. Specification testing can also be used to validate the accuracy of some production theory, such as Cobb-Douglas, CES, Translog, and related functious. There is a literature on specification testing for conventional regression models (see González-Manteiga and Crujeiras (2013) for a useful review). However, it would seem that there is as yet no analysis that discusses this problem for SFA models.

In this paper, we bridge the gap and test whether the production frontier 
function can be described by some known parametric function. To be precise, the null hypothesis is given as:

$$
H_{0}: m(X)=g\left(X, \beta_{0}\right)
$$

for some $\beta_{0}$ against the alternative hypothesis:

$$
H_{1}: m(X) \neq g(X, \beta)
$$

for any $\beta$, where $g(X, \beta)$ is a known smooth function with unknown $d$ dimensional parameter $\beta$.

Two test statistics, which are based on local smoothing and global smoothing, respectively, are proposed. To apply these two test statistics in practice, we suggest the residual-based wild bootstrap. A merit of our procedure is that, even under heteroscedasticity, the test statistics can still detect the alternative hypothesis efficiently. To the best of our knowledge, this is a novel contribution to the literature. The theory developed in this paper is useful for production mangers in their decisions on production (Tsekouras et al., 2017).

The remainder of the paper is organized as follows. In Section 2, we construct the test statistics and describe the residual-based wild bootstrap. In Section 3, simulation results are reported to examine the finite sample performance of the test statistics. An empirical application is given in Section 4 , and Section 5 concludes the paper. 


\section{Test Statistics}

To focus on specification testing of the production frontier function, we first discuss the estimation procedures for the parametric SFA model without specific distributional assumptions on $U$ and $V$.

\subsection{Estimation}

Let $\mu_{U}(X)=E(U \mid X), \epsilon=V-U+\mu_{U}(X)$ and $r_{1}(X)=Y-\epsilon$. Note that $E(\epsilon \mid X)=0$ always holds. We can then rewrite model (1) under the null hypothesis as follows:

$$
Y^{1}=Y+\mu_{U}(X)=g(X, \beta)+\epsilon .
$$

For the data set $\left(Y^{1}, X\right)$, the model is the traditional parametric regression model. If we can obtain the value of $\mu_{U}(X)$, then we can estimate the parameter $\beta$ by using nonlinear least squares based on $\left(Y^{1}, X\right)$. Thus, the most important and difficult part is how to estimate $\mu_{U}(X)$. To achieve this goal, we adopt the approach that is recently proposed by Simar et al. (2016).

Under the null hypothesis, model (1) can also be rewritten as:

$$
Y=r_{1}(X)+\epsilon
$$

where $E(\epsilon \mid X)=0$ still holds, which is the standard nonparametric regression model. We can obtain the estimator of $r_{1}(X), \hat{r}_{1}(X)$, by using nonparametric 
methods, such as kernels, local polynomials, and/or splines. Although there exist several nonparametric methods for regression models, in the following we focus on kernel-type estimators given by $\hat{r}_{1}(x)=\sum_{i=1}^{n} W_{n i}(x) Y_{i}$, with:

$$
W_{n i}(x)=\frac{K_{h}\left(x-X_{i}\right)}{\sum_{j=1}^{n} K_{h}\left(x-X_{j}\right)}
$$

and $K_{h}(\cdot)=K(\cdot / h) / h^{p}$, with $K(\cdot)$ the kernel function, and $h$ being the bandwidth.

Under the symmetry assumption on $V$, and the conditional independence of $U$ and $V$ given $X$, we have the following:

$$
\begin{aligned}
& E\left(\epsilon^{2} \mid X\right)=\operatorname{var}_{U}(X)+\operatorname{var}_{V}(X), \\
& E\left(\epsilon^{3} \mid X\right)=-E\left[\left(U-\mu_{U}(X)\right)^{3} \mid X\right],
\end{aligned}
$$

where $\operatorname{var}_{U}(X)$ and $\operatorname{var}_{V}(X)$ denote the conditional variances of $U$ and $V$ given $X$, respectively.

Denote $r_{j}(X)=E\left(\epsilon^{j} \mid X\right)$ for $j=2$ and 3. After estimation of $r_{1}(X)$, we can obtain the residuals, $\hat{\epsilon}=Y-\hat{r}_{1}(X)$. By adopting appropriate nonparametric techniques, we can estimate the functions $r_{j}(X)$ for $j=2$ and 3 consistently. Define:

$$
\hat{r}_{j}(x)=\sum_{i=1}^{n} W_{n i}(x)\left(Y-\hat{r}_{1}\left(X_{i}\right)\right)^{j},
$$

for $j=2$ and 3. Note that if $\mu_{U}(X)$ is a function of $E\left[\left(U-\mu_{U}(X)\right)^{3} \mid X\right]$, then we can easily estimate $\hat{r}_{3}(X)$. To achieve this goal, local parametric assumptions on the types of distributions of $U \mid x$ are necessary. 
Assume that $U|x \sim| N\left(0, \sigma_{U}^{2}(x)\right) \mid$ and that, conditionally on $X, U$ and $V$ are independent, which is the same paradigm as in Kumbhakar et al. (2007). As a result, we have:

$$
\begin{aligned}
\mu_{U}(X) & =E(U \mid X)=\sqrt{\frac{2}{\pi}} \sigma_{U}(X), \\
E\left(\epsilon^{2} \mid X\right) & =\frac{\pi-2}{\pi} \sigma_{U}^{2}(X)+\operatorname{var}_{V}(X), \\
E\left(\epsilon^{3} \mid X\right) & =\sqrt{\frac{2}{\pi}}\left(1-\frac{4}{\pi}\right) \sigma_{U}^{3}(X) \leq 0 .
\end{aligned}
$$

From the above equations, we can obtain the following :

$$
\begin{aligned}
& \hat{\sigma}_{U}(X)=\max \left\{0,\left[\sqrt{\frac{\pi}{2}}\left(\frac{\pi}{\pi-4}\right) \hat{E}\left(\epsilon^{3} \mid X\right)\right]^{1 / 3}\right\}, \\
& \hat{\mu}_{U}(X)=\sqrt{\frac{2}{\pi}} \hat{\sigma}_{U}(X) .
\end{aligned}
$$

(for further details, see Simar et al. (2016)).

After estimating $\hat{\mu}_{U}(X)$, we can estimate $\beta$ by using nonlinear least squares based on the data points, $\left\{\left(\hat{Y}_{i}^{1}, X_{i}\right) \mid i=1, \cdots, n\right\}$. Defining $\hat{Y}_{i}^{1}=$ $Y_{i}+\hat{\mu}_{U}\left(X_{i}\right)$, let $\epsilon_{0}=Y^{1}-g(X, \beta)$ to obtain the residuals under the null hypothesis, $\hat{\epsilon}_{0 i}=\hat{Y}_{i}^{1}-g\left(X_{i}, \hat{\beta}\right)$.

\subsection{Construction}

Under the null hypothesis, we can easily obtain:

$$
E\left(\epsilon_{0} \mid X\right)=E\left(Y+\mu_{U}(X)-g(X, \beta) \mid X\right)=E\left(g(X, \beta)+V-U+\mu_{U}(X)-g(X, \beta) \mid X\right)=0 .
$$


while under the alternative hypothesis, we obtain :

$$
\begin{aligned}
E\left(\epsilon_{0} \mid X\right) & =E\left(Y+\mu_{U}(X)-g(X, \beta) \mid X\right)=E\left(m(X)+V-U+\mu_{U}(X)-g(X, \beta) \mid X\right) \\
& =m(X)-g(X, \beta) \neq 0 .
\end{aligned}
$$

The above observations form the basis of the construction of the new test statistics. We introduce the local smoothing-based test statistic. Note that, under the null hypothesis, we have:

$$
E\left(\epsilon_{0} E\left(\epsilon_{0} \mid X\right) f(X)\right)=E\left[E^{2}\left(\epsilon_{0} \mid X\right) f(X)\right]=0,
$$

where $f(X)$ is the density function of $X$. Under the alternative hypothesis, the first term in the above equation must be positive. Thus, the empirical counterpart of this term can be used as the test statistic. By using the leaveone-out kernel estimator of $f(X)$ and $E\left(\epsilon_{0} \mid X\right)$, the following test statistic is constructed:

$$
T_{n 1}=\frac{1}{n(n-1)} \sum_{i=1}^{n} \sum_{j \neq i}^{n} K_{h}\left(X_{i}-X_{j}\right) \hat{\epsilon}_{0 i} \hat{\epsilon}_{0 j} .
$$

The type of test statistic given above is introduced in Zheng (1996), and proposed independently by Fan and Li (1996). In classical regression models, it can be shown that the distribution of $T_{n 1}$ converges to a centered normal as $n \rightarrow \infty$. However, we should note that, in the context of the SFA model, the asymptotic properties of $T_{n 1}$ can be complex due to the existence of the term $\mu_{U}(X)$. To formally study the asymptotic properties of $T_{n 1}$, we 
need to investigate the impact of the nonparametric estimation of $\hat{\mu}_{U}(X)$ on the estimation of $\beta$ explicitly. In this paper, we focus on investigating the numerical performance of $T_{n 1}$, and leave the theoretical project for future research.

We can construct an empirically-based test statistic. Note that, under the null hypothesis, the following equation holds:

$$
E\left(\epsilon_{0} I(X \leq x)\right)=0, \quad \forall x \in \mathbb{R}^{p}
$$

This motivates construction of the residual-based empirical process, as follows:

$$
R_{n}(x)=\frac{1}{\sqrt{n}} \sum_{i=1}^{n} \hat{\epsilon}_{0 i} I\left(X_{i} \leq x\right) .
$$

Then the Cramér-von Mises-type test statistic can be defined by:

$$
T_{n 2}=\int\left(R_{n}(x)\right)^{2} d F_{n}(x)
$$

where $F_{n}(x)$ is the empirical distribution based on $\left\{X_{1}, X_{2}, \cdots, X_{n}\right\}$.

Similarly, in classical regression models, it can be shown that the defined empirical process, $R_{n}(x)$ converges to a centered continuous Gaussian process, and the test statistic converges to the functional of this Gaussian process (see Stute (1997)), but the covariance function of the Gaussian process would be changed. We leave the formal theoretical analysis for future research. 
We follow the residual-based wild bootstrap method (see Stute et al., 1998) to determine whether to reject the null hypothesis using the following steps:

Step 1. Obtain $\hat{\mu}_{U}(X), \hat{\beta}$ and $\hat{\epsilon}_{0}$ by using the approach proposed in subsection 2.1 , and then construct $T_{n i}, i=1,2$, as in subsection 2.2 .

Step 2. Generate bootstrap observations, $Y_{i}^{*}=g\left(X_{i}, \hat{\beta}\right)-\hat{\mu}_{U}\left(X_{i}\right)+\hat{\epsilon}_{0 i} \times e_{i}$. Here $\left\{e_{i}\right\}_{i=1}^{n}$ is a sequence of i.i.d. random variables with zero mean, unit variance, and independent of the sequence $\left\{Y_{i}, X_{i}\right\}_{i=1}^{n}$. Usually, $\left\{e_{i}\right\}_{i=1}^{n}$ can be chosen to be i.i.d. Bernoulli variates with:

$$
P\left(e_{i}=\frac{1-\sqrt{5}}{2}\right)=\frac{1+\sqrt{5}}{2 \sqrt{5}}, \quad P\left(e_{i}=\frac{1+\sqrt{5}}{2}\right)=1-\frac{1+\sqrt{5}}{2 \sqrt{5}}
$$

Step 3. Let $T_{n i}^{*}, i=1,2$ be defined similarly as $T_{n i}, i=1,2$, based on the bootstrap sample, $\left\{Y_{i}^{*}, X_{i}\right\}_{i=1}^{n}$.

Step 4. Repeat Steps 2 and 3, B times, and calculate the $p$-value as $p_{i}^{B}=$ $\#\left\{T_{n i}^{*}>T_{n i}\right\} / B$

\section{Simulations}

We now perform simulations to examine the finite sample performance of the proposed test statistics. 


\section{Study 1}

$$
\begin{aligned}
& H_{11}: Y=5+5 X+a \exp \left\{X^{2}\right\}-U+V, \\
& H_{12}: Y=5+5 X+a \sin \{4 \pi X\}-U+V .
\end{aligned}
$$

The value $a=0$ corresponds to the null hypothesis, and $a \neq 0$ to the alternative. In the above models, we take $X \sim U(0,1), U \sim|N(0,1)|$, and $V \sim N\left(0, \sigma_{V}^{2}\right)$, where $\sigma_{V}=0.75 \times \sqrt{(\pi-2) / \pi}$. For the models, under the null hypothesis, $a=0$, this is Example 1 in Kumbhakar et al. (2007). For $H_{11}$, the sample size is taken to be 100 , and $a=\{0.0,0.3, \cdots, 1.5\}$ to examine the size and power performance of the proposed test statistics, $T_{n 1}$ and $T_{n 2}$. For $H_{12}$, we consider $n=50$ and 100 , and the sequence of $a$ is taken to be $a=\{0.0,0.2, \cdots, 1.0\}$

In the simulation study, the number of replications is 2,000. For each replication, $B=500$ bootstrapped samples are generated. In the nonparametric regression estimation, the kernel function is taken to be $K(u)=$ $15 / 16\left(1-u^{2}\right)^{2}$, if $|u| \leq 1$; and 0 , otherwise. The bandwidth is taken to be $h=\hat{\sigma}(X) \times n^{-1 / 5}$ for simplicity, where $\hat{\sigma}(X)$ is the empirical estimator of the standard deviation of $X$. The nominal level of $\alpha$ is set at 0.05 .

The simulation results are presented in Table 1. From the table, we have the following observations. First, for all situations considered, the empirical sizes of the two test statistics are all close to the nominal level. This 
implies that the proposed test statistics have accurate size. Second, when we consider empirical power, we can see clearly that the proposed tests are very sensitive to the alternative, such that, when the value of $a$ increases, power increases quickly. For model $H_{11}$, the second test statistic, $T_{n 2}$, can have higher power as compared with the first test statistic, $T_{n 1}$. However, for $H_{12}, T_{n 1}$ is more powerful. For model $H_{12}$, when the sample size is $n=100$, the power performance of both tests improves compared with sample size $n=50$.

\section{Study 2}

Consider the same models as in Study 1, but now introduce heteroscedasticity in the distribution of the technical inefficiency. Here, we have $U \mid X=$ $x \sim\left|N\left(0,(1+x)^{2}\right)\right|$. We should note that, under the null hypothesis, $a=0$, is Example 2 in Kumbhakar et al. (2007). This study investigates the impact of heteroscedasticity on the performance of the two proposed test statistics. Other settings are the same as in Study 1.

The simulation results are shown in Figure 1. For comparison, we also plot the simulation results of these two test statistics in Study 1. From this figure, we conclude that, compared with the results in Study 1, the powers of the two test statistics decrease significantly. This suggests that heteroscedasticity in the distribution of the technical inefficiency can have a negative impact on power performance. We can also see that, for $H_{11}$, 
$T_{n 2}$ performs better than $T_{n 1}$, while for $H_{12}, T_{n 1}$ is more powerful. These observations suggest that the two new test statistics should be viewed as complementary to each other.

\section{Empirical Application}

A rice production data set is available online, as described in the Preface of Coelli et al. (2005, p. xvi) (further details on the data can be found in Appendix 2 of Coelli et al. (2005)). The data set is recently analyzed in Wang et al. (2011) to calculate goodness-of-fit tests for the distribution of technical inefficiency. Here we use this data set to check whether the Cobb-Douglas model is plausible.

Following Coelli et al. (2005) and Wang et al. (2011), three inputs (area, labour and fertiliser) and one output (tonnes of freshly threshed rice) are used, and are denoted by $X=\left(X_{1}, X_{2}, X_{3}\right)=(A R E A, L A B O R, N P K)$, and $Y=P R O D$, respectively. The Cobb-Douglas model is given as follows:

$$
\ln Y=\beta_{0}+\sum_{i=1}^{3} \beta_{i} \ln X_{i}-U+V .
$$

In our context, the null hypothesis is:

$$
H_{0}: m(X)=\beta_{0}+\sum_{i=1}^{3} \beta_{i} \ln X_{i} .
$$

For sample size $n=344$, the values of $T_{n i}, i=1,2$, are 1.8062 and 616.5035, 
and the corresponding $p$-values are 0.160 and 0.774 , respectively. Therefore, for this data set, a Cobb-Douglas model is plausible.

\section{Concluding Remarks}

Though SFA models have been used widely in many disciplines, such as economics, finance and statistics, a formal specification testing procedure for the production frontier function has not yet been available. This paper develop two new test statistics by adopting local smoothing and global smoothing methods, respectively.

The asymptotic properties of the two test statistics under the null hypothesis, fixed alternative hypothesis, and local alternative hypothesis, have not been investigated. The existence of the inefficiency term, $U$, makes the analysis complicated. We leave these interesting and important theoretical studies to future research.

Without explicit asymptotic distributions under the null hypothesis, we have to rely on resampling approaches to calibrate the critical values. To this end, the residual-based wild bootstrap is suggested. The new proposed test statistics allow specification testing of the production frontier function, even under heteroscedasticity. The simulation studies showed that the sizes of the two test statistics are quite close to the nominal level, and that the 
powers are also satisfactory, even when the sample size is relatively small, at $n=50$. The theory developed in this paper is useful for production mangers in their decisions on production (Tsekouras et al., 2017).

\section{References}

[1] Aigner, D.J., Lovell, C.A.K. and Schmidt, P. (1977), Formulation and estimation of stochastic frontier models, Journal of Econometrics, 6, 21-37.

[2] Bonanno, G., De Giovanni, D., Domma, F. (2017), The 'wrong skewness' problem: a re-specification of stochastic frontiers, Journal of Productivity Analysis, 47(1), 49-64.

[3] Chen, Y.T. and Wang, H.J. (2012), Centered-residuals-based moment tests for stochastic frontier models, Econometric Reviews, 31, 625-653.

[4] Coelli, T.J. (1995), Estimators and hypothesis tests for a stochastic frontier function: a Monte Carlo analysis, Journal of Productivity Analysis, 6, 247-268.

[5] Coelli, T.J., Prasada Rao, D.S., Ó Donnell, C.J. and Battese, G.E. (2005). An Introduction to Efficiency and Productivity Analysis. 2nd Edition, Springer, New York. 
[6] Fan, Y. and Li, Q. (1996), Consistent model specification tests: Omitted variables and semiparametric functional forms, Econometrica, 64, 865890.

[7] Fan, Y., Li, Q. and Weersink, A. (1996), Semiparametric estimation of stochastic production frontier, Journal of Business and Economics Statistics, 14, 460-468.

[8] Fried, H., Lovell, C.A.K. and Schmidt, S. (2008), The Measurement of Productive Efficiency and Productivity Change, Oxford University Press, New York.

[9] González-Manteiga, W. and Crujeiras, R.M. (2013), An updated review of Goodness-of-Fit tests for regression models, Test, 22, 361-411.

[10] Greene, W.H. (1990), A Gamma-Distributed Stochastic Frontier Model, Journal of Econometrics, 46, 141-163.

[11] Greene, W.H. (2005), Reconsidering heterogeneity in panel data estimators of the stochastic frontier model Journal of Econometrics, 126(2), 269-303.

[12] Kopp, R.J., and Mullahy, J. (1990), Moment-based estimation and testing of stochastic frontier models, Journal of Econometrics, 46, 165-183. 
[13] Kumbhakar, S. and Lovell, C.A.K. (2000), Stochastic Frontier Analysis, Cambridge University Press, Cambridge.

[14] Kumbhakar, S.C., Park, B.U., Simar, L. and Tsionas, E.G. (2007), Nonparametric stochastic frontiers: A local likelihood approach, Journal of Econometrics, 137(1), 1-27.

[15] Lee, L.F. (1983), A test for distributional assumptions for the stochastic frontier functions, Journal of Econometrics, 22, 245-267.

[16] Meeusen, W., and Broeck, J.v.D. (1977), Efficiency estimation from Cobb-Douglas production functions with composed error, International Economic Review, 18(2), 435-444.

[17] Schmidt, P., and Lin, T.F. (1984), Simple tests of alternative specifications in stochastic frontier models, Journal of Econometrics, 24, 349-361.

[18] Simar, L., Van Keilegom, I. and Zelenyuk, V. (2016), Nonparametric least squares methods for stochastic frontier models. Journal of Productivity Analysis, in press.

[19] Simar, L. and Wilson, P. (2015), Statistical approaches for nonparametric frontier models: A guided tour, International Statistical Review, 83, $77-110$. 
[20] Stute, W. (1997), Nonparametric model checks for regression, Annals of Statistics, 25, 613-641.

[21] Stute, W., Gonzáles-Manteiga, W. and Presedo-Quindimil, M. (1998), Bootstrap approximation in model checks for regression, Journal of the American Statistical Association, 93, 141-149.

[22] Tsekouras, K., Chatzistamoulou, N., Kounetas, K. (2017), Productive performance, technology heterogeneity and hierarchies: Who to compare with whom, International Journal of Production Economics, 193, 465478.

[23] Wang, W.S., Amsler, C. and Schmidt, P. (2011), Goodness of fit tests in stochastic frontier models, Journal of Productivity Analysis, 35, 95-118.

[24] Zheng, J.X. (1996), A consistent test of functional form via nonparametric estimation techniques, Journal of Econometrics, 75, 263-289. 
Table 1: Simulated sizes and powers of proposed test statistics $T_{n 1}$ and $T_{n 2}$ for Study 1.

\begin{tabular}{|c|c|c|c|c|}
\hline$H_{11}$ & \multicolumn{4}{|c|}{$n=100$} \\
\hline$a$ & \multicolumn{3}{|c|}{$T_{n 1}$} & $T_{n 2}$ \\
\hline 0.0 & \multicolumn{3}{|c|}{0.0490} & 0.0530 \\
\hline 0.3 & \multicolumn{3}{|c|}{0.0730} & 0.0950 \\
\hline 0.6 & \multicolumn{3}{|c|}{0.1370} & 0.2370 \\
\hline 0.9 & \multicolumn{3}{|c|}{0.2685} & 0.4170 \\
\hline 1.2 & \multicolumn{3}{|c|}{0.4255} & 0.6430 \\
\hline 1.5 & \multicolumn{3}{|c|}{0.6445} & 0.8400 \\
\hline$H_{12}$ & \multicolumn{2}{|c|}{$n=50$} & \multicolumn{2}{|c|}{$n=100$} \\
\hline$a$ & $T_{n 1}$ & $T_{n 2}$ & $T_{n 1}$ & $T_{n 2}$ \\
\hline 0.0 & 0.0510 & 0.0480 & 0.0540 & 0.0450 \\
\hline 0.2 & 0.1240 & 0.0770 & 0.1920 & 0.1390 \\
\hline 0.4 & 0.3590 & 0.2100 & 0.7010 & 0.4280 \\
\hline 0.6 & 0.7190 & 0.4060 & 0.9640 & 0.8410 \\
\hline 0.8 & 0.9170 & 0.6880 & 0.9990 & 0.9840 \\
\hline 1.0 & 0.9790 & 0.8550 & 1.0000 & 0.9980 \\
\hline
\end{tabular}



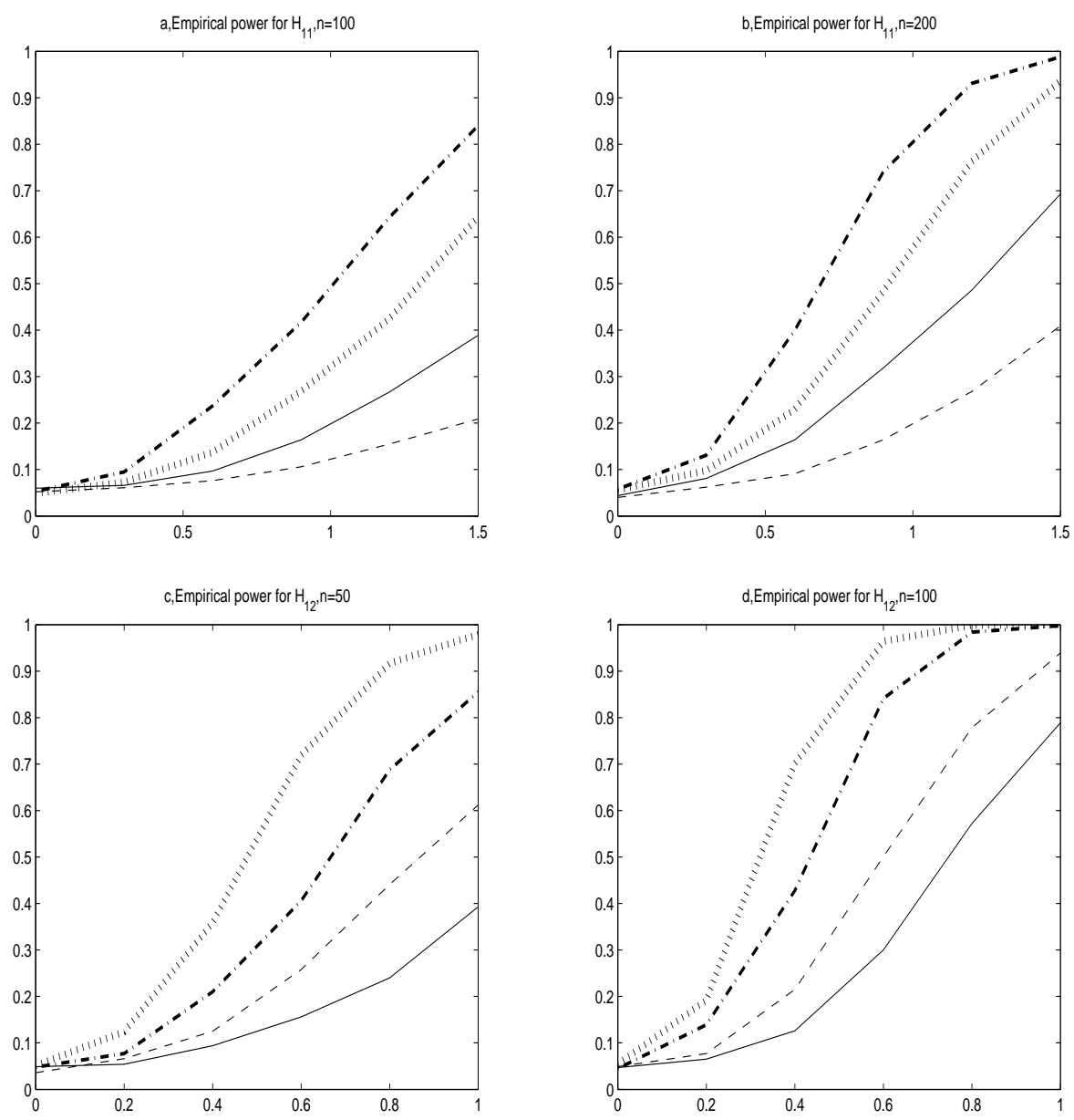

Figure 1: Powers of test statistics with $H_{11}$ and $n=100$ (top left corner), $H_{11}$ and $n=100$ (top right corner), $H_{12}$ and $n=50$ (lower left corner), and $H_{12}$ and $n=100$ (lower right corner), respectively. The dashed, dotted, solid and dot-dashed lines represent the results of $T_{n 1}$ for Study 2 and Study 1, and $T_{n 2}$ for Study 2 and Study 1, respectively. 